\title{
Opportunities and Challenges in Ultra-High Energy Resolution EELS
}

\author{
Ondrej L. Krivanek ${ }^{1}$, N. Dellby ${ }^{1}$, M.V. Hoffman ${ }^{1}$ and T.C. Lovejoy ${ }^{1}$ \\ ${ }^{1 .}$ Nion Co., 11511 NE $118^{\text {th }}$ St., Kirkland, WA 98034, USA
}

Two first observations of phonons by ultra-high energy resolution electron spectroscopy (UHERES) in the electron microscope were made two years ago [1,2], and much has been learned since. Opportunities and challenges remain aplenty, in instrumentation, theory, and applications.

UHERES has allowed the spatial distribution of phonons to be mapped [1], hydrogen to be detected by its vibrational signature $[3,4]$, and Rutherford scattering to be energy-analyzed in the electron microscope [5]. In the future, UHERES is likely to introduce further revolutionary capabilities such as determining, by aloof vibrational spectroscopy, the types of organic compounds attached to catalytic nanoparticles, and analyzing the molecular species present in biological tissues. Promising starts have been made in these directions, and will be reported in other contributions to the present session. This contribution focuses on the progress being made and principal challenges on the instrumentation side, and on our hopes for detecting an atomically resolved vibrational signal.

After progressing rapidly to about $10 \mathrm{meV}$ energy resolution (measured as full width at halfmaximum (FWHM) of the zero loss peak (ZLP)), the pace of further resolution improvements has slowed down, with the best resolution we have obtained equal to $8 \mathrm{meV}$. The slow-down is not surprising: the rapid gains came from a new technology we introduced (monochromating at ground potential [6]), and subsequent gains are necessarily more incremental. The situation is similar to the early days of successful aberration correction, in which rapid initial gains were followed by more incremental progress. The resemblance is not accidental: the energy resolution of both the monochromator and the electron energy loss spectrometer (EELS) is ultimately determined by how small a crossover each instrument can produce in its energy-dispersion plane. The crossover is an image of the electron source, broadened by diffraction, aberrations and instabilities. Just like with producing a small probe at the sample, making the monochromator and the spectrometer crossovers small is a question of opening up the illumination aperture (to decrease the diffraction limit) and controlling the aberrations and instabilities appropriately. $5 \mathrm{meV}$ and eventually $3 \mathrm{meV}$ resolution should be possible with our design. Reaching this level of performance will, however, require progress on several fronts, including improved aberration control in the monochromator and the spectrometer, and enhancing the stability of the whole microscope and especially of the spectrometer. We are working in these directions, and we will report on our progress at the meeting.

Another major concern is the magnitude of the beam current that can be used. Monochromating a $300 \mathrm{meV}$ wide electron beam with an energy-selecting slit open to $10 \mathrm{meV}$ removes $97 \%$ of the beam current, i.e. a $300 \mathrm{pA}$ beam is reduced to $10 \mathrm{pA}$. We use slit widths as narrow as 3-5 meV, which typically result in beam currents of 3-5 pA. 30-100 pA would be much more useful for mapping experiments. The long-term solution most likely lies in developing ultra-bright cold fieldemission guns (CFEGs). Once more, such a development is not expected to happen overnight.

Fig. 1 shows the theoretically possible probe size, as a function of the slit width and the desired probe current for a $100 \mathrm{keV}$ monochromated STEM with CFEG giving a coherent current of $100 \mathrm{pA}$ 
and $0.35 \mathrm{eV}$ energy width, and a STEM probe corrected to $50 \mathrm{mrad}$ half-angle. Making the slit narrower reduces the beam current and this can be compensated, up to a certain extent, by demagnifying the source less, thus maintaining the beam current while improving the energy resolution and worsening the spatial resolution. Note that due to several different factors limiting the energy resolution when the slit is nearly closed, the slit width needed for $10 \mathrm{meV}$ EELS resolution is about $5 \mathrm{meV}$, but when the slit is much wider, the attainable energy resolution is roughly equal to the slit width. Two useful compromise settings have been marked: operating with a $140 \mathrm{pm}, \sim 10 \mathrm{meV}$ wide probe (slit width $=5 \mathrm{meV}$ ) that contains $10 \mathrm{pA}$ probe current $\mathrm{I}_{\mathrm{p}}$ (orange diamond), and with a $60 \mathrm{pm}, 100 \mathrm{meV}$ probe with $\mathrm{I}_{\mathrm{p}}=10 \mathrm{pA}$ (blue diamond). The deep sub- $\AA$ spatial resolution is made possible by the probe half-angle of $50 \mathrm{mrad}$ and the fact that monochromation makes the chromatic aberration of the probe-forming optics less important.

The $140 \mathrm{pm}, 10 \mathrm{pA}, \sim 10 \mathrm{meV}$ EELS performance can be reached with our prototype EELS. It should allow vibrational spectra to be acquired at atomic resolution, and there is theoretical consensus that non-dipole (impact) scattering produces an atomically sharp signal [7,8]. We have not yet detected this signal, most likely for two reasons. First, dipole scattering, which dominates at large interaction distances (and which can be used for damage-free aloof beam vibrational spectroscopy [3]) is relatively strong, and liable to mask the localized signal. Second, the spatially localized signal is delocalized in reciprocal space, and its efficient collection requires combining 30 mrad or greater EELS collection half-angle with $10 \mathrm{meV}$ and better energy resolution. This is a lot to ask from an EELS, and we are working on achieving this kind of performance on a regular basis.

\section{References}

1. O.L. Krivanek et al., Nature 514 (2014) 209.

2. T. Miata et al., Microscopy 63 (2014) 377.

3. P. Rez et al., Nature Communications, in print (2016).

4. P.A. Crozier et al., Microsc. Microanal. 21 (Suppl 3, 2015) 1473.

5. T.C. Lovejoy et al., Microsc. Microanal. 20 (Suppl 3, 2014) 558.

6. O.L. Krivanek et al., Microscopy 62 (2013) 3.

7. P. Rez, Microsc. Microanal. 20 (2014) 671-677.

8. C. Dwyer, Phys Rev B 89 (2014) 054103.

Figure 1. Theoretical probe size vs. probe current for different slit widths of our UHERES system at $100 \mathrm{kV}$ primary voltage. The $\mathrm{X}$-axis scale shown at the top refers to the solid curve, the bottom scale to all the curves. The upper (orange) highlighted point has been demonstrated experimentally, while the lower one (blue) is a work in progress.

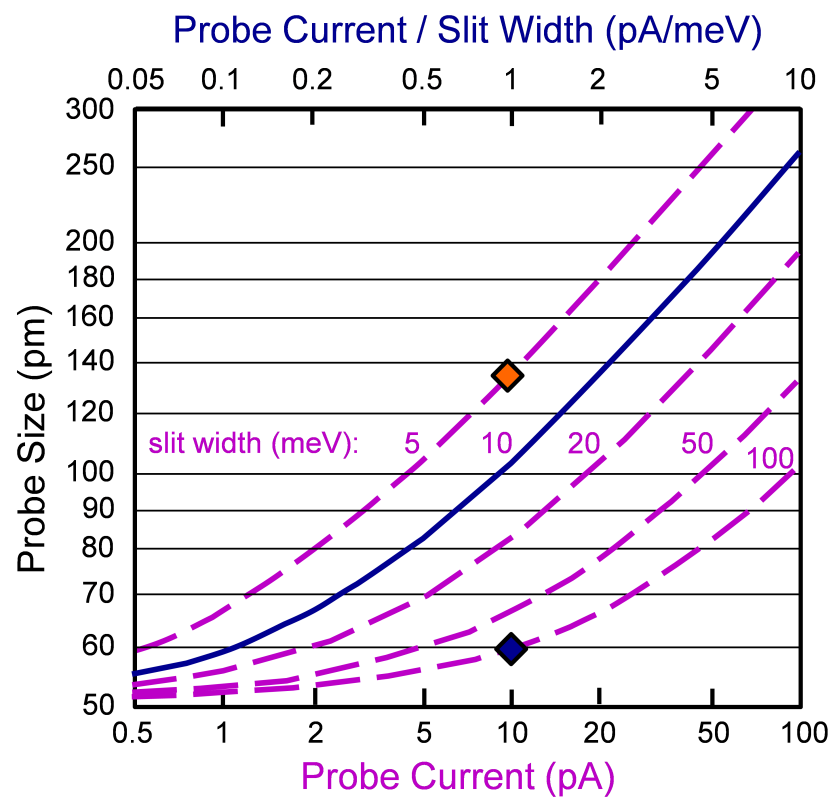

